Paola Miki Higa ${ }^{1}$ Mónica Valdivieso Vargas-Machuca $^{2}$

${ }^{1}$ Cirujano Dentista.

Docente del Departamento Académico de Estomatología del Niño y el Adolescente.

Facultad de Estomatología. Universidad Peruana Cayetano Heredia

\section{Características cráneo-faciales en pacientes con síndrome de Down en dos colegios de educación especial en Lima}

Miki P, Valdivieso M. Características cráneo-faciales en pacientes con síndrome de Down en dos colegios de educación especial en Lima. Rev Estomatol Herediana 2004;14(1-2) : 51 - 53.

\section{RESUMEN}

Para plantear un correcto plan de tratamiento dental para pacientes con síndrome de Down, necesitamos conocer mejor las características más prevalentes de esta entidad. El objetivo de este estudio fue establecer las características cráneo-faciales de pacientes con síndrome de Down de dos colegios de educación especial de Lima. Las características evaluadas fueron la forma de cráneo, forma de cara, perfil vertical, perfil anteroposterior, musculatura facial, y características de labios, lengua y paladar duro. Se realizaron historias clínicas odontológicas a una población total de 99 pacientes inscritos en los colegios que participaron en el estudio. La muestra final de 22 pacientes se obtuvo por saturación, ya que comprendió a todos los pacientes con síndrome de Down evaluados. La edad promedio fue 12 años. El diagnóstico médico de cada paciente fue proporcionado por los padres. Se realizó la prueba univariada de distribución de frecuencias para establecer la relación entre las variables dependientes y la independiente. Los resultados mostraron una alta prevalencia de mesocefalia, musculatura facial normotónica, perfil antero-posterior tipo recto, perfil vertical normodivergente, incompetencia labial y paladar duro profundo.

Palabras clave: SÍNDROME DE DOWN / CEFALOMETRÍA / CARA.

\section{Craneo-facial characteristics of patients with Down's syndrome}

ABSTRACT

In order to build an appropriate dental treatment plan for patiens with Down's syndrome, we need to learn more about their most prevalent characteristics. The aim of this study was to describe the craneo-facial characteristics of patients with Down's syndrome of two special education schools in Lima, Perú. The characteristics evaluated in this study were head shape, facial type, vertical profile, anteroposterior profile, facial musculature and lips, tongue and hard palate. Clinical examinations were made to a total of 99 patients enrolled at the schools participating in this study. Only 22 patients presented a medical diagnosis of Down's Syndrome, and were included on this study. The mean age was 12 years. The medical diagnosis were supplied by the parents during the evaluation. The statistical test of frequency was used to establish a relationship between the variables. The results showed a high frecuency of mesocephalic head type, normotonic musculature, straight anteroposterior profile, normodivergent vertical profile, labial incompetence, and deep hard palate.

Keywords : DOWN SYNDROME / CEPHALOMETRY / FACE.

\section{Introducción}

En las últimas décadas, la población mundial de personas mentalmente discapacitadas ha aumentado considerablemente. Esto se debe principalmente a los adelantos en las áreas de neonatología y pediatría, que permiten salvar a bebés que anteriormente no habrían sobrevivido. Este aumento paulatino de la población de pacientes especiales lleva a una mayor demanda de tratamientos dentales dirigidos a dichos pacientes (1).
Los pacientes discapacitados, ya sea física o mentalmente, requieren de un especial cuidado de la salud oral por parte de los familiares y odontólogos, ya que muchas veces la disminución de sus facultades dificulta o imposibilita un autocuidado. A esto se suma la mayor prevalencia de ciertas patologías orales como enfermedad periodontal, lesiones de tejidos blandos, maloclusión y problemas articulares. El tratamiento de las maloclusiones es importante para restablecer el correcto funcionamiento del sistema ortognático (2).

Son muchos los factores que contribuyen a la mayor prevalencia de maloclusiones en pacientes mentalmente discapacitados. Entre estos encontramos deformidades craneofaciales, alteraciones de crecimiento y desarrollo, postura anormal de la lengua, alteraciones neuromusculares y alteraciones dentales. Estas malformaciones generan a la vez una serie de disfunciones en el habla, la masticación, la deglución, y la respiración (2). 
La importancia de este estudio radica en la necesidad de conocer las características cráneo-faciales de mayor prevalencia en los pacientes con síndrome de Down, para poder plantear un correcto tratamiento integral que permita mejorar las condiciones de vida de dichos pacientes.

\section{Material y método}

El presente estudio fue de tipo transversal y descriptivo. Se realizaron historias clínicas odontológicas a 99 pacientes mentalmente discapacitados matriculados en dos colegios de educación especial de Lima. Los diagnósticos médicos fueron obtenidos por referencia de los padres durante la anamnesis. La muestra final de 22 pacientes se obtuvo por saturación, que correspondió a todos los pacientes con síndrome de Down con historia clínica completa. La edad promedio fue de 12 años. El diagnóstico médico de síndrome de Down se considero como variable independiente para los análisis estadísticos. Las variables dependientes evaluadas fueron la forma del cráneo, forma de la cara, perfil anteroposterior, perfil vertical, musculatura facial y alteraciones en labios, lengua y paladar duro. Se realizó la prueba univariada de distribución de frecuencias para establecer la relación entre las variables dependientes y la variable independiente.

\section{Resultados}

La tabla 1 muestra la distribución de pacientes según género.

La tabla 2 muestra los resultados en relación al número y porcentaje de las variables analizadas en este estudio.

\section{Discusión}

No se han encontrado estudios similares que establezcan la frecuencia de

Tabla 1. Distribución de la muestra según género

\begin{tabular}{|lccc|}
\hline & M & F & Total \\
\hline S. Down & $17(77.3 \%)$ & $5(22.7 \%)$ & $22(100.0 \%)$ \\
\hline
\end{tabular}

Tabla 2. Frecuencia de las características cráneo-faciales en pacientes con síndrome de Down.

\begin{tabular}{|c|c|c|c|}
\hline & Característica & $\mathbf{n}$ & $\%$ \\
\hline Forma del cráneo & $\begin{array}{l}\text { Mesocéfalo } \\
\text { Braquicéfalo } \\
\text { Dolicocéfalo }\end{array}$ & $\begin{array}{l}11 \\
6 \\
5\end{array}$ & $\begin{array}{l}50.00 \\
27.27 \\
22.23\end{array}$ \\
\hline Forma de la cara & $\begin{array}{l}\text { Braquifacial } \\
\text { Mesofacial } \\
\text { Dolicofacial } \\
\end{array}$ & $\begin{array}{l}9 \\
7 \\
6 \\
\end{array}$ & $\begin{array}{l}40.90 \\
31.81 \\
27.27 \\
\end{array}$ \\
\hline Perfil anteroposterior & $\begin{array}{l}\text { Recto } \\
\text { Convexo } \\
\text { Cóncavo } \\
\end{array}$ & $\begin{array}{l}11 \\
6 \\
4\end{array}$ & $\begin{array}{l}50.00 \\
27.27 \\
18.18 \\
\end{array}$ \\
\hline Perfil vertical & $\begin{array}{l}\text { Normodivergente } \\
\text { Hipodivergente } \\
\text { Hiperdivergente }\end{array}$ & $\begin{array}{l}16 \\
3 \\
3\end{array}$ & $\begin{array}{l}72.74 \\
13.63 \\
13.63\end{array}$ \\
\hline Musculatura facial & $\begin{array}{l}\text { Normotónico } \\
\text { Hipotónico } \\
\text { Hipertónico }\end{array}$ & $\begin{array}{l}12 \\
8 \\
1\end{array}$ & $\begin{array}{l}54.55 \\
36.36 \\
4.55\end{array}$ \\
\hline Labios & $\begin{array}{l}\text { Incompetentes } \\
\text { Competentes }\end{array}$ & $\begin{array}{l}13 \\
9\end{array}$ & $\begin{array}{l}59.09 \\
40.91\end{array}$ \\
\hline Lengua (tamaño) & $\begin{array}{l}\text { Macroglosia } \\
\text { Tamaño normal } \\
\end{array}$ & $\begin{array}{l}11 \\
11 \\
\end{array}$ & $\begin{array}{l}50.00 \\
50.00 \\
\end{array}$ \\
\hline Lengua (textura) & $\begin{array}{l}\text { Textura normal } \\
\text { Lengua escrotal }\end{array}$ & $\begin{array}{l}18 \\
4 \\
\end{array}$ & $\begin{array}{l}81.82 \\
18.18 \\
\end{array}$ \\
\hline Paladar duro & $\begin{array}{l}\text { Profundo } \\
\text { Normal }\end{array}$ & $\begin{array}{l}20 \\
2\end{array}$ & $\begin{array}{l}90.10 \\
9.10\end{array}$ \\
\hline
\end{tabular}

todas las variables analizadas en este estudio. Muchos autores mencionan ciertas características como descripción general de los pacientes con síndrome de Down, pero no presentan valores estadísticos o estudios que los respalden.

Con respecto a la forma de cráneo, Fiske y Shafik (3); y Regezi (4) en revisiones bibliográficas describieron a los pacientes con síndrome de Down como braquicefálicos, sin mostrar valores estadísticos que sustenten dicha afirmación. Esto no concuerda con los resultados obtenidos en este estudio, donde se observó una mayor prevalencia de forma de cráneo mesocefálica. Solo un $27.27 \%$ de la muestra presentó cráneo braquicefálico.

John L. Down (5) en 1887, en la primera descripción de pacientes con el síndrome que hoy lleva su nombre, mencionó que dichos pacientes presentan una forma de cara generalmente ancha. Nuestros resultados están de acuerdo con dicha afirmación, ya que se observó una frecuencia de forma braquifacial de $40.92 \%$.

Con relación al tipo de perfil ánteroposterior, Down (5) describió una marcada tendencia a un perfil recto o plano. Gandy (6), en una base de datos sobre múltiples enfermedades pediátricas el 90\% de pacientes con síndrome de Down presentaban un perfil ántero-posterior tipo recto. Los resultados hallados en este estudio mostraron solo un porcentaje de $50 \%$.

Machuca et al. (2), en una revisión bibliográfica afirman que existe una alta prevalencia de maloclusiones en pacientes con retardo mental, debido a diversas alteraciones entre las que se encuentran alteraciones neuromusculares como hipotonía o hipertonía. Fiske y Shafik (3); Sindoor y Fayetteville (7); y Sindoor y Flanagan (8) describen una alta frecuencia de hipotonía muscular entre los pacientes con síndrome de Down. Gandy (6) también indicó que el 80\% presentaba hipotonía muscular. Los resultados de este trabajo mostraron una menor prevalencia de hipotonía muscular, con un porcentaje de $36.36 \%$.

Sindoor y Fayetteville (7); Sindoor 
y Flanagan (8); y Fiske y Shafik (3) afirman que los pacientes con síndrome de Down presentan incompetencia labial. Esto concuerda con nuestros resultados que mostraron una frecuencia de 59.09\% de incompetencia labial.

Según la descripción hecha por John L. Down (5), los pacientes con síndrome de Down presentan lengua larga y gruesa, con una superficie mucho más rugosa de lo normal. Fiske y Shafik (3); Sindoor y Fayetteville (7) y Sindoor y Flanagan (8) en revisiones bibliográficas también afirman que existe una mayor tendencia a presentar macroglosia y lengua escrotal en pacientes con síndrome de Down. Pirela, Salazar y Manzano (1) observaron que el $100 \%$ de pacientes con síndrome de Down presentaban lengua escrotal y macroglosia. Gandy (6) afirma que el 60\% presenta una macroglosia relativa, y 37\% presenta lengua escrotal. En el presente estudio, se observó una alta prevalencia macroglosia relativa. La característica de lengua escrotal se observa en un menor porcentaje (18.18\%).

Sindoor y Fayetteville (7) en una revisión bibliográfica afirman que los pacientes con síndrome de Down presentan una disminución en el largo y ancho; y un aumento en la profundidad del paladar duro debido al pobre desarrollo del tercio medio facial. Los resul- tados de este estudio están de acuerdo con la descripción de los autores, ya que se observó una frecuencia de paladar profundo de $90.9 \%$.

El análisis de los resultados del presente estudios nos permite afirmar que en los pacientes con síndrome de Down existe una alta prevalencia de forma de cara braquifacial, hipotonicidad muscular, perfil antero-posterior tipo recto, incompetencia labial, macroglosia relativa, lengua escrotal y paladar duro profundo. Asi mismo consideramos necesario realizar más estudios que presenten datos estadísticos confiables sobre las características craneofaciales predominantes en pacientes con síndrome de Down.

\section{Referencias bibliográficas}

1. Pirela MA, Salazar CR, Manzano M. Patología bucal prevalente en niños excepcionales. Acta Odontológica Venezolana 1999; 37 (3): 193-98.

2. Machuca C, Martínez F, Machuca G, López L, Bullón P. Tratamiento ortodóntico en los distintos niveles de prevención en pacientes con necesidades especiales. Av Odontoestomatol 1999; 15(4): 577-81.

3. Fiske J, Shafik H. Down's syndrome and oral care. J South African Dent Assoc 2001; 56 (9): 407-15.

4. Regezi J. Patología bucal. 1ra. Ed.
México DF: McGraw-Hill Interamericana, 1991:471.

5. Down JL. Observations on an ethnic classification of idiots. The Journal of Mental Science 1867; 13: 121-3.

6. Gandy A, Pediatric Database (PEDBASE) Homepage. 1994 www.icondata.com/health/pedbase/ index.htm

7. Sindoor D, Fayetteville N. Down syndrome. Oral Surg Oral Med Oral Pathol Oral Radiol Endod 1997; 84 (3): 279-85.

8. Sindoor D, Flanagan T. Orthodontic considerations in individuals with Down syndrome: A case report. The Angle Orthodontist 1999; 69(1): 8588.

9. Nelson. Tratado de Pediatría. 16ta Ed. México DF: McGraw-Hill Interamericana, 2001:138-140.

10. Rodríguez C, Bratos E, Garcillan R, Rioboo R. Estudio epidemiológico en adultos discapacitados psíquicos: Parte III. Maloclusión y otras anomalías dentarias. Av Odontoestomatol 2000; 16(9):527-534.

\section{Dirección de Correspondencia}

Miki Paola Higa

Los Melones 364 - Lima 12, Perú

435-3556/9930-4540

paolamiki@terra.com.pe 